\title{
Automated search for Galactic star clusters in large multiband surveys
}

\section{Discovery of 15 new open clusters in the Galactic anticenter region $\star$}

\author{
S. E. Koposov ${ }^{1,2,3}$, E. V. Glushkova ${ }^{2}$, and I. Yu. Zolotukhin ${ }^{2}$ \\ 1 Max Planck Institute for Astronomy, Königstuhl 17, 69117, Heidelberg, Germany \\ e-mail: koposov@mpia.de \\ 2 Sternberg Astronomical Institute, Universitetskiy pr. 13, 119992 Moscow, Russia \\ e-mail: elena@sai.msu.ru \\ 3 Institute of Astronomy, University of Cambridge, Madingley Road, Cambridge CB3 OHA, UK
}

Received 7 September 2007 / Accepted 29 April 2008

\section{ABSTRACT}

\begin{abstract}
Aims. According to some estimations, there are as many as 100000 open clusters in the Galaxy, but less than 2000 of them have been discovered, measured, and cataloged. We plan to undertake data mining of multiwavelength surveys to find new star clusters.

Methods. We have developed a new method to search automatically for star clusters in very large stellar catalogs, which is based on convolution with density functions. We have applied this method to a subset of the Two Micron All Sky Survey catalog toward the Galactic anticenter.

We also developed a method to verify whether detected stellar groups are real star clusters, which tests whether the stars that form the spatial density peak also fall onto a single isochrone in the color-magnitude diagram. By fitting an isochrone to the data, we estimate at the same time the main physical parameters of a cluster: age, distance, color excess.

Results. For the present paper, we carried out a detailed analysis of 88 overdensity peaks detected in a field of $16 \times 16$ degrees near the Galactic anticenter. From this analysis, 15 overdensities were confirmed to be new open clusters and the physical and structural parameters were determined for 12 of them; 10 of them were previously suspected to be open clusters by Kronberger (2006) and Froebrich (2007). The properties were also determined for 13 yet-unstudied known open clusters, thus almost tripling the sample of open clusters with studied parameters in the anticenter. The parameters determined with this method showed a good agreement with published data for a set of well-known clusters.
\end{abstract}

Key words. Galaxy: structure - open clusters and associations: general - surveys - catalogs

\section{Introduction}

Star clusters are unique laboratories for investigation of a wide range of astrophysical problems relating to star formation, stellar evolution, the formation and structure of the Milky Way, and the distance scale of the Universe. As star clusters are usually single-age and single-metallicity populations, distance, age, and reddening in the cluster's direction can be determined with much higher accuracy than for isolated, or "field", stars. To define at least a reliable ranking of the open cluster properties, we need a large sample of objects whose age, distance, and metallicity are accurately and homogeneously known. So far, 1756 open clusters were cataloged (Dias et al. 2002), but the basic physical parameters are known for less than 700 objects. And, all these parameters were derived by different authors based on heterogeneous observational data. Most of the open clusters in the Galaxy are probably not yet found because open clusters are concentrated near the Galactic plane where extinction by interstellar dust is most severe. Some literature estimates put the total number of open clusters in the Galaxy at $10^{5}$ (see, for example Surdin 2000). Modern all-sky surveys (e.g., Two Micron All Sky Survey (2MASS), Deep Near Infrared Survey of the Southern

\footnotetext{
* Figures 7-18 and Table 3 are only available in electronic form at http: //www . aanda.org
}

Sky (DENIS), Sloan Digital Sky Survey (SDSS), etc.) provide a large store of information to study open clusters comprehensively and homogeneously. Near-infrared surveys are especially useful because the data are far less affected by high reddening in the Galactic plane where the most open clusters are located.

Numerous attempts were made in recent years to search for star clusters using such large surveys. However, the total number of newly-discovered clusters with robust determinations of their physical parameters does not exceed two dozen. Dutra et al. (2003) performed a visual search for IR clusters and similar objects in the direction of known nebulae using the 2MASS Atlas and found 179 embedded clusters and stellar groups. However, it proved impossible to find the physical parameters of this type of objects through isochrone fitting. Ivanov et al. (2002) and Borissova et al. (2003) found 11 peaks by automated algorithm and 3 peaks by visual inspection in the apparent stellar surface density in the 2MASS point source catalog. They detected mostly embedded IR clusters, so the physical parameters could be derived only for one object. Drake (2005) performed an automated search for clusters in the United States Naval Observatory (USNO) A2 catalog using the method developed by Ivanov et al. (2002) and found 8 new candidates. However, their basic parameters were not derived. Kronberger et al. (2006) visually inspected Digitized Sky Survey (DSS) and 2MASS images and 
selected 66 candidate clusters. For 9 of 24 of the most probable clusters within this sample, the authors determined fundamental parameters by isochrone fitting. Froebrich et al. (2007) used star density maps obtained from 2MASS and found 1021 new cluster candidates. The authors statistically evaluated the contamination of their sample to be of about $50 \%$ and left verification of the nature of each individual cluster for future investigations.

We developed a new efficient method of searching stellar catalogs for star clusters of different radii based on the convolution of the cataloged stellar source density maps with Gaussians (a similar method was used to search for dwarf Spheroidal galaxies and globular clusters in SDSS; Koposov S. et al. 2007; Koposov et al. 2008). The method automatically finds cluster candidates and then confirms them by testing whether the spatially clustered, potential cluster members lie on the same isochrone in the color-magnitude diagram. At the same time, this procedure determines the basic cluster parameters (age, radius, distance, and color excess) by fitting the isochrone position. Below, we describe our method of automatic search for stellar overdensities and the results of its application to the 2MASS data in the field of $16 \times 16$ degrees in the region of the Galactic anticenter.

\section{Method of automated search for star clusters}

This work aims to provide a fast, simple, and efficient method of identifying open clusters in very large photometric catalogs, such as 2MASS, SDSS, DENIS etc. The overall density of the stars in the Milky Way (MW) is high at low latitudes and can vary rapidly because of dust, etc. So, it is not easy to find star clusters algorithmically on such a complex background. Even when a peak is found, it is important to check whether the candidate is indeed an evolutionally connected group of cluster members or merely a group of stars clustered by chance.

Therefore, the method must be capable of detecting density peaks on a sharply changing background and to evaluate their statistical significance. To develop a universal technique for all data sources, we have to make it independent of any pixelization effects and thus applicable to star structures of any size. To ensure this, we built a density map, which presents the number of counts in (RA, Dec) coordinates. Then, we convolved this image with a special filter demonstrated in Fig. 1. The filter curve is the difference between two 2-D Gaussian profiles and has zero integral. Employing this special shape of filter, we ensure that a flat, or even slowly changing background produces a zero signal, whereas the concentrations of stars exhibit a high signal. The family of such filters called Difference of Gaussians are well studied and used in computer vision science for feature detection at various scales (Babaud et al. 1986; Lindenberg 1998). The convolution with this filter is equivalent to the subtraction of the density maps convolved with the gaussians of different widths. The density map convolved with the small Gaussian is used to detect the small scale overdensities, while the density map convolved with wider Gaussian is the estimation of local background.

The following formulae demonstrate our convolution procedure. First, we obtain the distribution of stars on the sky $M(\mathrm{RA}, \mathrm{Dec})$ :

$M(\mathrm{RA}, \mathrm{Dec})=\sum_{i} \delta(\mathrm{RA}, \mathrm{Dec})$

Then, this map is convolved with the filter:

$M(\mathrm{RA}, \mathrm{Dec})=M(\mathrm{RA}, \mathrm{Dec}) *\left(G\left(\mathrm{RA}, \mathrm{Dec}, \sigma_{1}\right)-G\left(\mathrm{RA}, \mathrm{Dec}, \sigma_{2}\right)\right)$

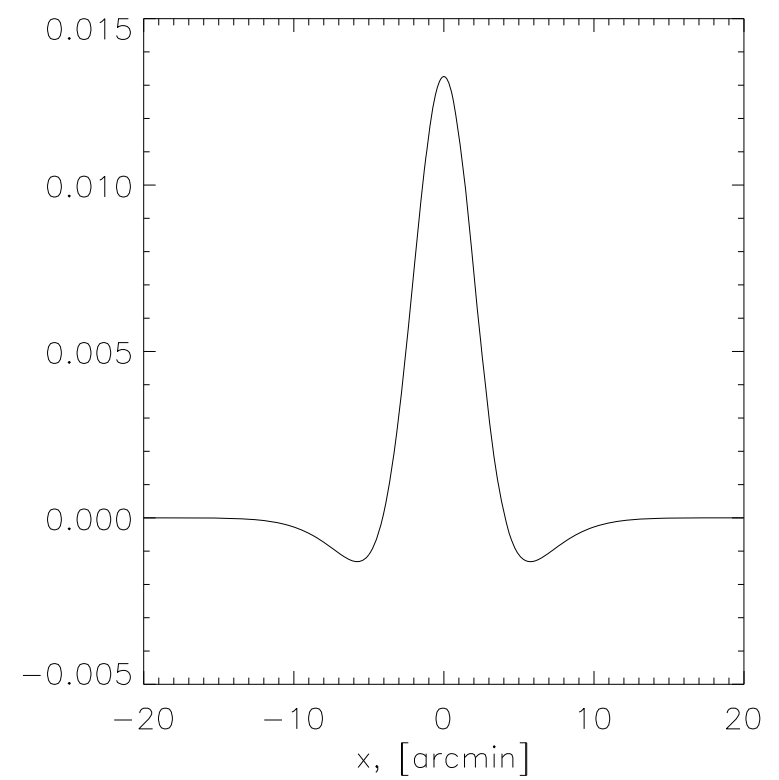

Fig. 1. The one-dimensional slice of the $2 \mathrm{D}$ filter which we used for the convolution for $\sigma_{1}=3^{\prime}$ and $\sigma_{2}=6^{\prime}$.

where $G(\mathrm{RA}, \mathrm{Dec}, \sigma)$ is the circular 2D Gaussian with unity integral and width of $\sigma$. On the last step, we normalize the convolved map to get the statistical significances of all density fluctuations:

$S(\mathrm{RA}, \mathrm{Dec})=$

$\sqrt{4 \pi} \sigma_{1} \frac{M(\mathrm{RA}, \mathrm{Dec}) *\left(G\left(\mathrm{RA}, \mathrm{Dec}, \sigma_{1}\right)-G\left(\mathrm{RA}, \mathrm{Dec}, \sigma_{2}\right)\right)}{\left.\sqrt{M(\mathrm{RA}}, \mathrm{Dec}) * G\left(\mathrm{RA}, \mathrm{Dec}, \sigma_{2}\right)\right)}$.

That map $S(\mathrm{RA}, \mathrm{Dec})$ shows the deviation of $M(\mathrm{RA}, \mathrm{Dec}) *$ $G\left(\mathrm{RA}, \mathrm{Dec}, \sigma_{1}\right)$ above the background estimate given by $M(\mathrm{RA}, \mathrm{Dec}) * G\left(\mathrm{RA}\right.$, Dec, $\left.\sigma_{2}\right)$. Under the assumption of Poisson distribution of sources and $\sigma_{2} \gg \sigma_{1}$, the $S$ (RA, Dec) should be normally distributed with variance of 1 . The detection of overdensities on this map is very easy and may be done by simple thresholding, i.e., to select all the overdensities more statistically significant than $5 \sigma$, we need find all the pixels on the map having $S$ (RA, Dec) $>5$. We typically used the detection threshold of 4.5 sigmas.

Note that for clusters of the size close to that of the inner Gaussian, the filter is very close to optimal. Also, it is important to understand that the choice of $\sigma_{2}$ is related to the scale on which the background estimate is obtained. Ideally, $\sigma_{2}$ should be rather large and much larger than the $\sigma_{1}$, but unfortunately the 2MASS data in the MW plane suffers significantly from extinction and the density of stars varies on very small scales. Therefore, we are forced to use the $\sigma_{2}$, which is not much larger than $\sigma_{1}$, to have a more local estimate of the background. See also Koposov et al. (2008) for a discussion of the method.

The example of the convolved image from the 2 MASS point source catalog for the $16^{\circ} \times 16^{\circ}$ anticenter region of our Galaxy is shown in Fig. 2. A large population of peaks is clearly seen. As we show below, most of these peaks can be attributed to open clusters.

After the density peaks are detected, each individual peak should be examined as to whether it relates to a real cluster or just a random fluctuation. To answer this question, we built the Hess-diagram representing the spatial density of stars on a color-magnitude diagram (CMD) (The Hess-diagrams used here are actually the difference of the $2 \mathrm{D}$ histogram of colormagnitude of stars inside the circle around the overdensity 


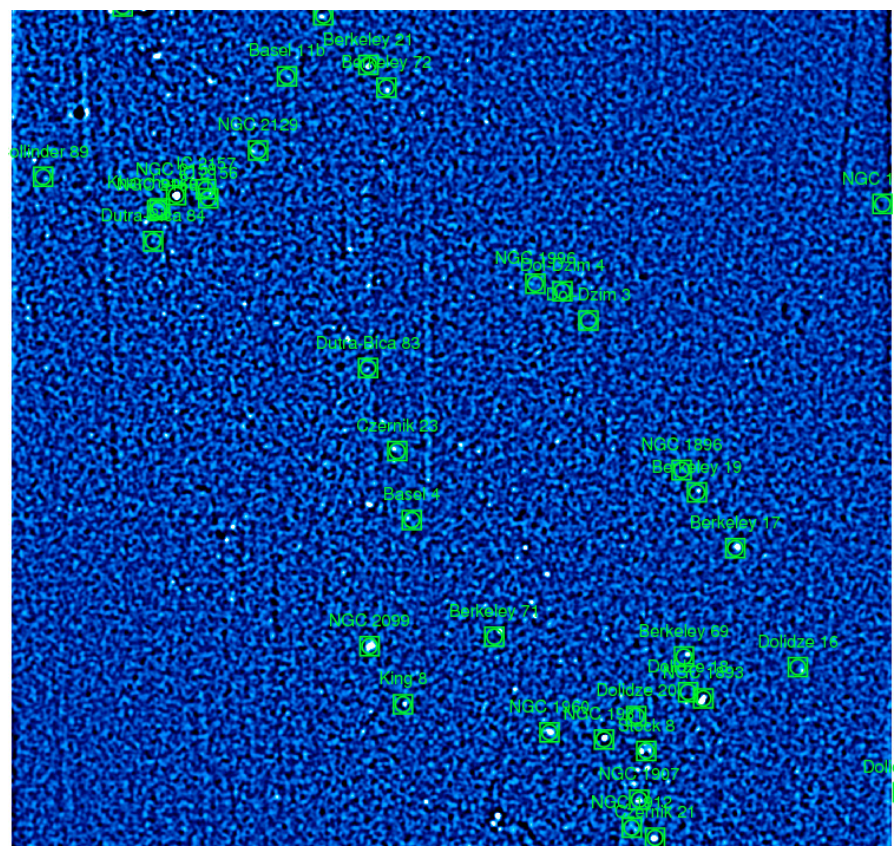

Fig. 2. The $16^{\circ} \times 16^{\circ}$ map of the overdensities in the anticenter region from 2MASS PSC. The known open clusters from the Dias catalog (Dias et al. 2002) are marked.

center (cluster) and the 2D histogram of color-magnitude of stars in the annulus outside the circle (field)). As rule, a real cluster is seen on this Hess-diagram by its main sequence and sometimes, by a red giant branch. If we detected cluster on the Hess-diagram, we fitted its CMD with the isochrone of solar metallicity taken from Girardi et al. (2002). To do this, we automatically shifted the isochrone along the coordinate axes with steps equal to $1 / 100$ of the full range of ordinates and abscissas. At every step, we varied the isochrone age in the interval of $\log ($ age $)$ from 6.60 to 10.25 with the step of 0.05 . At each step, for each isochrone age, we automatically built the radial density distribution for stars lying in the vicinity of the isochrone (the distance in the color index is less than 0.05 - assumed cluster members) and for all other stars (the distance is greater than 0.05 - supposed field stars). Ideally, the distribution function for field stars should be flat whereas the cluster members should feature a noticeable concentration toward the center. In practice, the radial density distribution of field stars shows a weak central concentration because of the number of unresolved binary stars - cluster members fall into this category (see, for example, Fig. 5) - or because of the poor photometry. That is why to qualify the radial density distribution, we calculated the contrast ratio in the following manner: we divided the value of the peak to the mean plateau for each distribution, then we found the ratio of the two values obtained. For example, in Fig. 5 the contrast is $(4.9 / 1) /(2.7 / 2)=3.63$. We believe that the best position of the isochrone on the color-magnitude diagram corresponds to the maximum contrast ratio at radial density distribution. Testing our technique for well-studied open clusters, we found that the contrast ratio should be greater than 2 . By fitting the position of the isochrone to obtain the maximum contrast ratio on the density plot, we simultaneously found main physical parameters of a cluster: age, distance, and color excess. If all plots (Hess-diagram, CMD, and the radial density distribution) verified the reality of the cluster, then the overdensity under study was considered to be a real cluster. Thus, we developed a method of an automated search for stellar overdensities and proposed a reliable criterion for verification of these overdensities as star clusters.

\section{Application of the method to the 2MASS data}

The 2MASS survey (Skrutskie et al. 2006) gives us a comprehensive dataset both to search for new open clusters and to test our method: it covers $99.998 \%$ of the sky with uniform precise photometry and astrometry in the $J(1.25 \mu) ; H(1.65 \mu)$; and $K_{\mathrm{S}}(2.16 \mu)$ photometric bands. The global 2MASS sensitivity is 15.8 for $J$-band; 15.1 for $H$-band; and 14.3 for $K_{\mathrm{S}}$ at $S / N=10$. For this reason, we investigated the $(J, J-H)$ color-magnitude diagrams in our work, but also used $\left(K_{\mathrm{s}}, J-K_{\mathrm{s}}\right)$ diagrams to confirm found cluster parameters and be sure that the relation $E(J-H) / E\left(J-K_{\mathrm{s}}\right)$ agrees with the normal extinction law.

To extract $J, H, K_{\mathrm{s}}$ photometry and astrometry data, we used the Virtual Observatory resource named Sternberg Astronomical Institute Catalogue Access Services (SAI CAS; http://vo. astronet.ru), allowing us to access the largest astronomical catalogs (Koposov S. E. et al. 2007). For our purposes, we only selected the stars that have the quality flags better than $U$ in each filter $J, H, K_{\mathrm{s}}$.

Our primary goal was to detect clusters that have diameters from few to ten arcminutes, so we used $\sigma_{1}=3^{\prime}$ and $\sigma_{2}=6^{\prime}$ in the filter function. Other than the richest ones, clusters having diameters more than 15-20 arcmin do not show as a rule the visual overdensity on the sky. Such extended clusters are usually found by common proper motions or radial velocities.

As a first application, we studied a field of 16 by 16 degrees towards the Galactic anticenter and detected 88 density peaks of $>4.5 \times \sigma$ significance. We compared these cluster candidates with open clusters listed by Dias et al. (2002) (in practice, we used the online version of Dias' catalog at http: //www . astro. iag.usp.br/ wilton/); 23 of our significant peaks can be matched to known, optically-visible clusters. Furthermore, we matched 9 density peaks to embedded infrared clusters from the list created by Bica et al. (2003a,b). Dias' catalog contains an additional 15 open clusters in this region, but our method does not detect them. Six of 15 clusters are not reliable clusters: they are not found in the DSS and 2MASS images, three of them are doubtful clusters according to Dias et al. (2002), one such object has no entry in the WEBDA database on open clusters developed by Paunzen and Mermilliod (http://www. univie.ac. at/webda/). Another 5 clusters having diameters ranging from 20 to 60 arcmin according to Dias et al. (2002), are considered to be clusters due to the common pattern of the proper motion of star members, and only 2 clusters among them, NGC 1912 and NGC 2168, exhibit stellar overdensities. The remaining four clusters have density peaks under $4.5 \times \sigma$ significance, and three of them are seen on 2MASS images as weak embedded clusters. These have no available data, except diameters, in Dias' catalog. Because more than half of the known clusters, that are on our list of detected density peaks have unreliable or no parameter measurements, we completed the detailed analysis of all 88 peaks, including known clusters.

We built the Hess-diagram in $(J, J-H)$ coordinates. We plotted CMD within radius $r$ with the value between 2 and 7 arcmin (depending on the cluster size) around the overdensity center, then we subtracted the CMD for field stars built in the ring between the two radii: $3 \times r$ and $4 \times r$. Each CMD was previously normalized to the number of stars and smoothed using a 3-pixel Gaussian. Figure 3 displays the Hess-diagram for the new open cluster Koposov 52 built within the radius of 4 arcmin around the cluster center (left-hand side) and CMD for field stars 

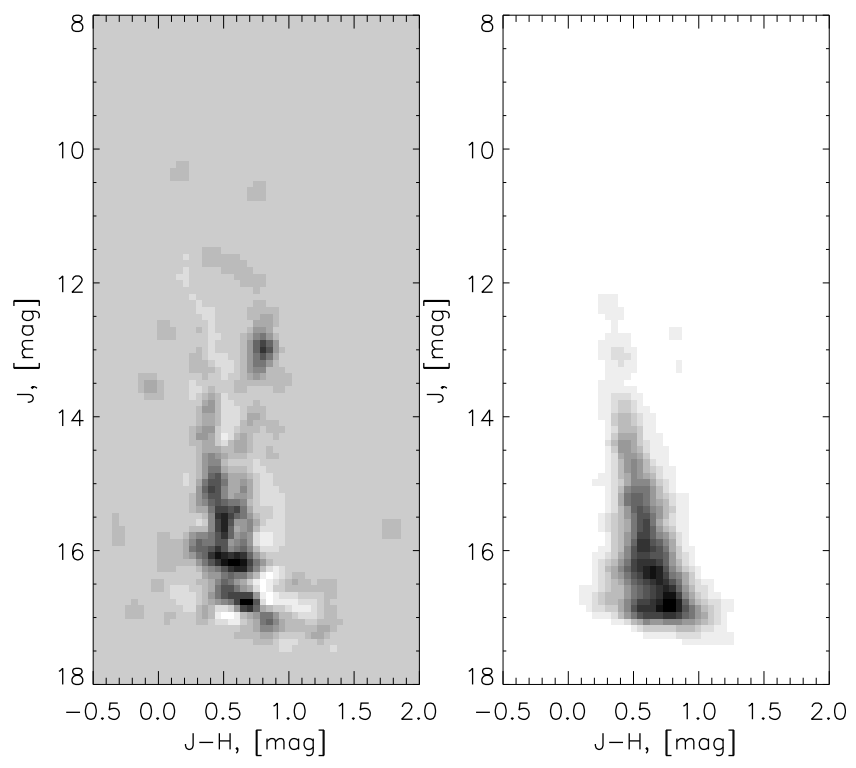

Fig. 3. Hess-diagrams for open cluster Koposov 52. The left panel shows the Hess diagram of the central $4^{\prime}$ of the cluster with subtracted Hess diagram of the background. The right panel shows the Hess diagram of the background stars.

in the ring around the cluster (right-hand side). The cluster can clearly be seen on the Hess-diagram by its main sequence and red clump stars.

If a cluster-suspect manifested itself at the Hess-diagram, then we fitted its CMD with an isochrone, by Girardi et al. (2002) of solar metallicity and simultaneously verified whether the detected stellar group was a real star cluster by plotting radial density distribution for stars lying on the isochrone and for all other stars (see for details Sect. 2). The fitted isochrone with the age of $\log (t)=8.95$ for Koposov 52 is shown in Fig. 4. Star members are taken within the radius of 2 arcmin around its center; the position of the isochrone leads to the following estimations: $E(J-H)=0.34$ and $(m-M)_{J}=13.20$. The radial density distribution corresponding to this fitted isochrone is displayed in Fig. 5: solid circles denote the stars deviating from the isochrone by less than $0.05 \mathrm{mag}$ in color $(J-H)$; open circles denote all other stars. Therefore, the total density profile of all stars is the sum of the open circle profile and solid circle profile. The errors plotted on the datapoints are simple Poisson errors. The ratio of contrasts for the "isochrone" and "field" stars calculated in Sect. 2 equals to 3.63. It is important to note that the background value (value at large radii) for the solid circle profile is much lower than the background value for the empty circle profile. This illustrates the advantage of using the isochrone filter for the detection and analysis of overdensities - with such a filter, overdensity is much more obvious due to the reduced background.

We performed isochrone fitting on $(J, J-H)$ diagram because there is a higher magnitude limit for $J$-band in 2MASS, and used a 15-arcmin region around stellar overdensity. We independently performed the same fitting procedure on a $\left(K_{\mathrm{s}}, J-K_{\mathrm{s}}\right)$ $\mathrm{CMD}$ and compared the distances obtained from two fittings with each other, and the relation $E(J-H) / E\left(J-K_{\mathrm{s}}\right)$ with the normal extinction law given by Cardelli et al. (1989), which equals to 0.55 . Also we used the relations $A_{K_{\mathrm{s}}}=0.670 \times E\left(J-K_{\mathrm{s}}\right)$, $A_{J}=0.276 \times A_{V}$, and $E(J-H)=0.33 \times E(B-V)$ from the paper by Dutra et al. (2002).

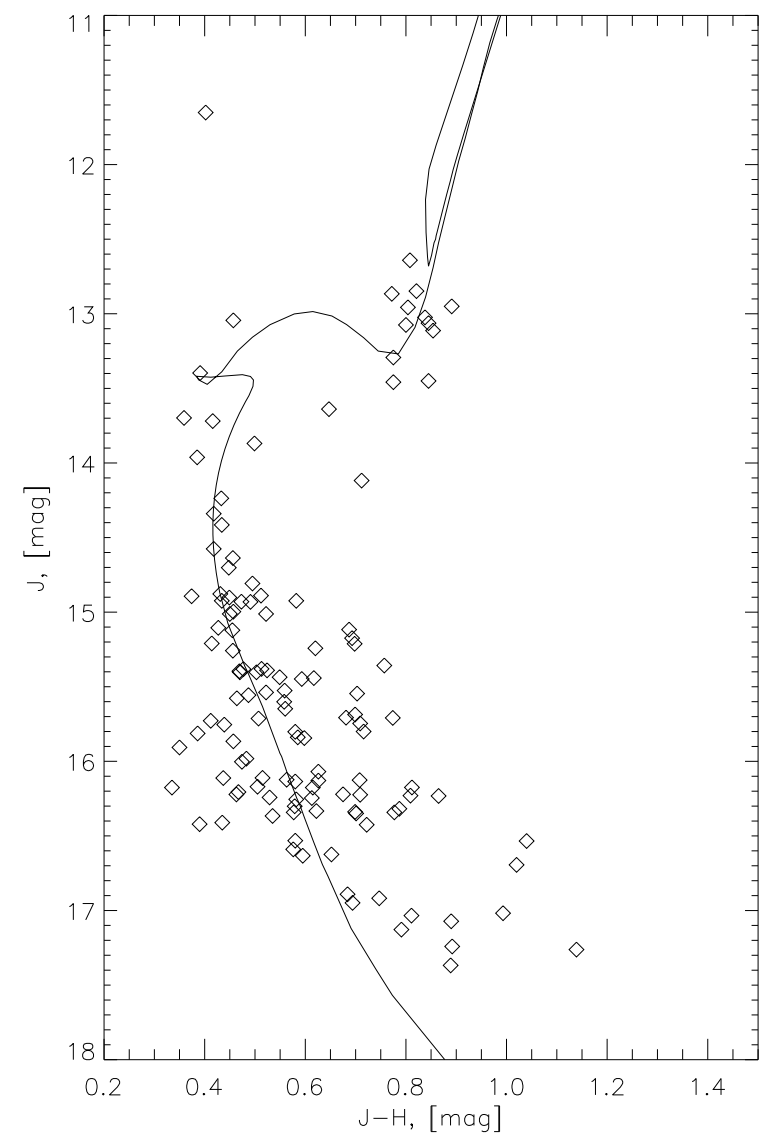

Fig. 4. Color-magnitude diagram for cluster Koposov 52 inside the radius of 2 arcmin. The fitted isochrone with $\log (t)=8.95, E(B-V)=$ $0.04,(m-M)_{0}=12.32$ is overplotted.

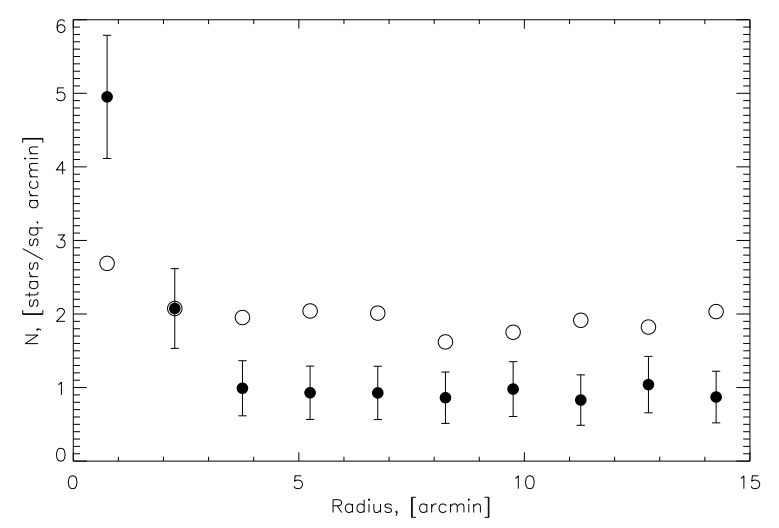

Fig. 5. Radial density distribution for stars in the field of Koposov 52. Solid circles represent the density of the stars lying closely to the isochrone. Open circles denote the density of the field stars lying far from the isochrone.

The cluster was considered to be a real cluster, if all plots (Hess-diagram, CMD, and the radial density distribution) verified the reality of the cluster.

\section{Results}

We used the routine described above to study all 88 overdensities. We found that 11 stellar overdensities turn out to be new, optically-visible clusters. The parameters for all these clusters are listed in Table 1. One of them, Koposov 52, was published earlier as KSE18 (Koposov et al. 2005; Zolotukhin et al. 2006) 
Table 1. Parameters of new clusters.

\begin{tabular}{|c|c|c|c|c|c|c|c|c|c|}
\hline Name & Other name & $\begin{array}{c}\text { RA (J2000) } \\
\text { h:m:s }\end{array}$ & $\begin{array}{c}\text { Dec }(\mathrm{J} 2000) \\
\text { d:m:s }\end{array}$ & $\begin{array}{c}D \\
\operatorname{arcmin}\end{array}$ & $\begin{array}{c}E(B-V) \\
\text { mag }\end{array}$ & $\frac{E(J-H)}{E(J-K)}$ & $\begin{array}{c}(m-M)_{0} \\
\mathrm{mag}\end{array}$ & $\begin{array}{c}\text { Distance } \\
\text { pc }\end{array}$ & $\begin{array}{c}\text { Age } \\
\log (\mathrm{yr})\end{array}$ \\
\hline Koposov 10 & FSR 795 & $05: 47: 28.6$ & $+35: 25: 56$ & 4 & $0.81 \pm 0.25$ & 0.79 & $11.54 \pm 0.3$ & $2000 \pm 300$ & $<8.6$ \\
\hline Koposov 12 & FSR 802 & 06:00:56.2 & $+35: 16: 36$ & 9 & $0.30 \pm 0.03$ & 0.51 & $11.55 \pm 0.03$ & $2050 \pm 50$ & $8.90 \pm 0.1$ \\
\hline Koposov 27 & Teutsch 1 & $05: 39: 30.0$ & $+33: 21: 00$ & 3 & $0.45 \pm 0.1$ & 0.69 & $12.8 \pm 0.5$ & $3700 \pm 900$ & $<8.65$ \\
\hline Koposov 36 & & $05: 36: 50.6$ & $+31: 12: 39$ & 9 & $0.83 \pm 0.11$ & 0.63 & $11.16 \pm 0.16$ & $1700 \pm 150$ & $<8.35$ \\
\hline Koposov 43 & FSR 848 & $05: 52: 14.6$ & $+29: 55: 09$ & 8 & $0.38 \pm 0.10$ & 0.44 & $12.21 \pm 0.09$ & $2800 \pm 120$ & $9.30 \pm 0.1$ \\
\hline Koposov 49 & Teutsch 10 & $05: 44: 22.2$ & $+28: 49: 13$ & 6 & $0.42 \pm 0.05$ & 0.94 & $11.46 \pm 0.22$ & $2000 \pm 200$ & $<9.15$ \\
\hline Koposov 52 & Teutsch 5 & $05: 53: 48.9$ & $+26: 50: 26$ & 5 & $1.03 \pm 0.04$ & 0.58 & $12.32 \pm 0.11$ & $2900 \pm 140$ & $8.95 \pm 0.1$ \\
\hline Koposov 53 & & 06:08:56.2 & $+26: 15: 49$ & 3 & $0.34 \pm 0.04$ & 0.61 & $12.52 \pm 0.03$ & $3200 \pm 100$ & $<8.5$ \\
\hline Koposov 62 & & 06:18:02.0 & $+24: 42: 38$ & 6 & $0.34 \pm 0.02$ & 0.57 & $12.21 \pm 0.05$ & $2800 \pm 60$ & $9.40 \pm 0.1$ \\
\hline Koposov 63 & FSR 869 & 06:10:01.7 & $+24: 33: 38$ & 5 & $0.26 \pm 0.04$ & 0.40 & $12.32 \pm 0.28$ & $3000 \pm 350$ & $9.15 \pm 0.1$ \\
\hline Koposov 77 & & $05: 43: 52.3$ & $+21: 42: 37$ & 5 & $0.57 \pm 0.01$ & 0.55 & $11.23 \pm 0.02$ & $1750 \pm 50$ & $9.65 \pm 0.1$ \\
\hline
\end{tabular}

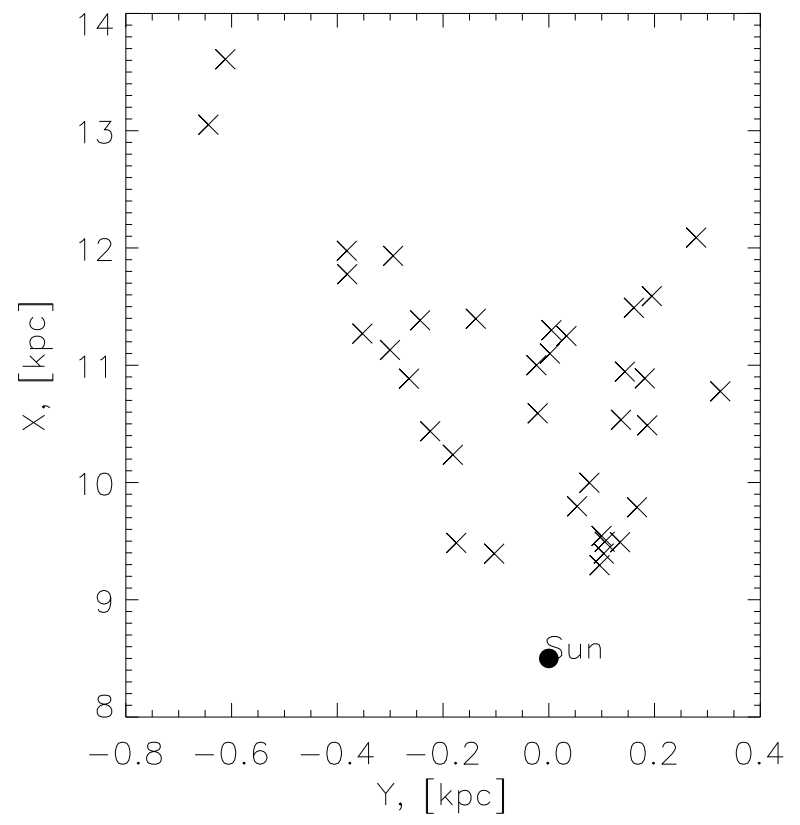

Fig. 6. Distribution of clusters across the galactic plane.

and then independently found by Kronberger et al. (2006) as Teutsch 51. Four other clusters coincide with cluster candidates from the list compiled by Froebrich et al. (2007), and two clusters coincide with stellar agglomerations reported by Kronberger et al. (2006). Note that in both papers cited, these objects are considered as possible clusters with futher investigation necessary to clarify their nature. In our work, we not only discovered these clusters by an independent method, but also verified their nature and found their fundamental parameters. That is why we consider them as new clusters.

Note that we are not able to make a precise estimation of the age of the young clusters, if their color-magnitude diagrams do not exhibit red or blue giants or supergiants. Provided that the positions of an isochrone with respect to the axes of coordinates is unchanged, we can alter its age within a broad range (in some cases, up to 8.20 in $\log ($ age $)$ ) without having a noticeable effect on the contrast on the radial-density distribution plot. This can be attributed to the existence of an extended vertical section of $(J, J-H)$ and $\left(K_{\mathrm{s}}, J-K_{\mathrm{s}}\right)$ isochrones and the absence of massive stars in poor clusters. Therefore, we were only able to make the upper boundary for the age estimation of Koposov 36 and Koposov 53, Koposov 10, Koposov 27, and Koposov 49 clusters. While it is possible that some of the bright stars on the CMD are giants belonging the cluster, there is no statistically meaningful way to check that with only photometric data, therefore we assign only the upper limits for the ages of these 5 clusters.

The errors in color excess, distance moduli, distances and ages are evaluated from the differences in the parameters derived from isochrones fitted in $(J, J-H)$ and $\left(K_{\mathrm{s}}, J-K_{\mathrm{s}}\right)$ diagrams. Hess-diagrams, radial density distribution, and fitted isochrone in $(J, J-H)$ CMD's for 10 new clusters (except for Koposov 52) are given in Figs. 7-16 in the Appendix. Four of the 11 new clusters are relatively young, less than $100 \mathrm{Myr}$, whereas the other clusters in this set are very old, exceeding 1 Gyr. Distances of all clusters from the Sun are ranged between 1.5 and $3.5 \mathrm{kpc}$.

Also, we found 4 new infrared clusters embedded in the nebulae, which are similar to the clusters found by Bica et al. (2003a) and Bica et al. (2003b): their coordinates are presented in Table 2, and Hess-diagrams are shown in Figs. 17, 18 in the Appendix. For IR clusters, the cluster reveals itself as a cloud on the left-hand diagram; the effect of the differential extinction is also clearly noticeable. The right-hand diagram displays CMD of field stars around the cluster. Because of a high value of reddening, it was impossible to fit isochrones and find parameters for these clusters, except Koposov 41. The Hess-diagram for this cluster exhibits the main sequence, and we can fit it with the isochrone of the age of $4 \mathrm{Myr}$. This best-fit isochrone was used to find the distance of $2200 \mathrm{pc}$ and the color excess of $E(B-V)=1.95$.

Thirty two overdensities turned out to be known clusters: 23 were matched to the objects from the Dias catalog (Dias et al. 2002), and 9, to IR clusters from the list by Bica et al. (2003a) and Bica et al. (2003b).

In Table 3, we present the data for all clusters from the catalog compiled by Dias et al. (2002), that were detected by our technique within the square region studied. Although two clusters, NGC 1912 and NGC 2168, were not detected because of their large diameters (about 25 arcmin), we added their parameters to the table, as they exhibit slight overdensities and can be studied by our methods. In some cases, we obtained more precise coordinates of the center of clusters, so we give new coordinates for all clusters.

In the columns, we give parameters of the clusters both listed in the Dias catalog (Dias et al. 2002) or in WEBDA database and measured by applying our methodology to the 2MASS catalog. For clusters Be 72, Cz 21, Cz 23, Cz 24, DC 8, Pis 27, Dias does not publish any parameters except their coordinates and diameters. However, in the WEBDA database there are data on Be 71 taken from the paper by Lata et al. (2004): $E(B-V)=0.85$, $d=3900 \mathrm{pc}, \log (t)=8.80$. We observe that the color excess and the age well agree with our parameters, whereas the 
Table 2. Coordinates of new infrared embedded clusters.

\begin{tabular}{llccc}
\hline \hline Name & Other name & $\begin{array}{c}\text { RA (J2000) } \\
\text { h:m:s }\end{array}$ & $\begin{array}{c}\text { Dec (J2000) } \\
\text { d:m:s }\end{array}$ & $\begin{array}{c}D \\
\text { arcmin }\end{array}$ \\
\hline Koposov 7 & FSR 784 & $05: 40: 44.1$ & $+35: 55: 25$ & 6 \\
Koposov 41 & FSR 839 & $06: 03: 58.0$ & $+30: 15: 41$ & 4 \\
Koposov 58 & FSR 849 & $05: 51: 11.0$ & $+25: 46: 41$ & 2 \\
Koposov 82 & Teutsch 136 & $06: 11: 55.8$ & $+20: 40: 14$ & 4 \\
\hline
\end{tabular}

distance exceeds our value by $1500 \mathrm{pc}$. The difference in distance evaluations (when the age estimations are pretty close) can be attributed to the fact that the authors fitted $(V, B-V)$ CMD by Zero Age Main Sequence (ZAMS) given by SchmidtKaler (1982) to estimate the distance modulus, and the theoretical isochrones given by Girardi et al. (2002) to find the cluster's age, while we used 2MASS theoretical isochrones by Girardi et al. (2002) to evaluate all cluster's parameters.

Parameters we measured for 7 clusters (Be 19, Be 71, Kronberger 1, King 8, NGC 1931, Stock 8 and NGC 2158) significantly differ from the data in the catalog by Dias et al. (2002) and in WEBDA, but we suppose our results are more precise and homogeneous. Parameters of Be 19 were obtained by Christian (1980) as following: employing the UBV photographic photometry data, the author compared the CMD of Be 19 with both the color-magnitude diagram of NGC 752 and the theoretical isochrones by Ciardullo (1979). Parameters of King 8 cluster, which appeared in the catalog by Dias et al. (2002) were calculated by Loktin et al. (2001) using photographic photometry by Christian (1981). However, Christian (1981) published the distance to the clusters equal to $3.5 \mathrm{kpc}$, which essentially differs from the value of 6403 pc by Loktin et al. (2001). Parameters of Stock 8 were also obtained by Loktin et al. (2001) using the UBV photometry of 32 stars only measured by different authors; these stars do not show any sequence on CMD. As for cluster Kronberger 1, the isochrone corresponding to the parameters available in the Dias et al. (2002) catalog fitted the main sequence of field stars, whereas the cluster members are noticeably shifted to the right in the $(J, J-H)$ CMD because of extinction. The effect of extinction is clearly seen on the corresponding Hess-diagrams. Parameters of NGC 1931 cluster were obtained by Loktin et al. (2001) using published photometry data by Bhatt et al. (1994), but the authors themselves give the distance of $2170 \mathrm{pc}$. This cluster is embedded in a nebula, so its Hess-diagram shows a slightly scattered area occupied by the cluster. We fitted its CMD with a very young isochrone, and the distance appeared to be three times smaller than that by Dias et al. (2002). The estimations of the distance to NGC 2158 cluster differ from each other very noticeably (by $2 \mathrm{kpc}$ ). Dias et al. (2002) take data from Loktin et al. (2001), who used compilative data and automatic method to find the cluster's parameters. At the same time, our data are in good accordance with parameters by Carraro et al. (2002) who obtained 3600 pc for the distance, $E(B-V)=0.55$, and $\log (t)=9.3$ by fitting isochrone from Girardi et al. (2000) and then comparing the best-fit with the simulated synthetic CMD. The discussion above demonstrates that the authors often publish controversial estimations of the cluster parameters even if they employ the same observational data. Although our estimations are sometimes based on less deep photometric data, they have an essential advantage in homogeneity of both observational data and used isochrones and fitting methods.

Members of NGC 1893 cluster detected on the Hess-diagram do not lie on the same isochrone, so we cannot find a satisfactory fitting. This fact can be reasonably attributed to the existence of pre-main sequence stars in this cluster described by Vallenari et al. (1999). The distance to the cluster found by Vallenari et al. (1999) equals $4300 \mathrm{pc}$ (cf. with $6000 \mathrm{pc}$ in the Dias catalog).

In the remaining 11 cases, our parameters closely agree (to within the error levels) with the corresponding data available from the catalog by Dias et al. (2002). These error levels are as follow: $200-500 \mathrm{pc}$ for the distances, $0.10^{\mathrm{m}}$ for the color excess $E(B-V)$, and 0.05 for $\log (t)$.

There are five additional clusters that have parameters in Dias' catalog and that were not detected in the field under study (see Sect. 3). However, the distances and other parameters for four of them were obtained from very poor $(V, B-V)$ diagrams, which do not show any sequence or clump at the CMD, and we did not consider these clusters.

At the end, 41 of 88 density peaks showed no evidence of being real clusters. In these cases, we detect either a nebula, or a bright star, or occasional groups of stars, whose density for some reasons exceeds the density of field stars.

In Fig. 6, we also show the distribution of all clusters under investigation across the galactic plane: both the newlyopened clusters and the known ones, whose parameters were (re)determined in the present study. The crosses indicate the clusters with $\log ($ age $)$ less then 8.00; all young clusters are situated from 1 to $3.5 \mathrm{kpc}$ from the Sun.

We do not quantify the detection efficiency of our algorithm. In the square under our study in the vicinity of the Galactic anticenter, we detect as much as $95 \%$ of clusters listed with the diameters less than 15 arcmin in the Dias et al. (2002) catalog. But ideally the efficiency can be determined only with simulations, since none of existing catalags is complete and free of selection effects. The detailed analysis of the detection efficiency and selection effects of the algorithm is left for our future work.

\section{Conclusions}

We have demonstrated the new method of searching for star clusters in the data from large surveys and applied it to the 2MASS data. In the small field of 16 by 16 degrees in the poor region of Galactic anticenter, we have found and verified 15 new open clusters. Ten of them coincide with cluster candidates from papers by Froebrich et al. (2007) and Kronberger et al. (2006). However, we not only discovered these objects independently, but also investigated their nature. We developed an automated method, which involves three different techniques: Hessdiagram, color-magnitude diagram in $(J, J-H)$ and $\left(K_{\mathrm{s}}, J-K_{\mathrm{s}}\right)$, and radial density distribution. For 12 of the new clusters, we obtained main physical parameters: ages, distances, and color excesses. We also used our methods to evaluate the same physical parameters for all known clusters detected in the selected area. We found that out of 25 such clusters, only in 11 cases we can accept the values of the cluster parameters listed in the catalog by Dias et al. (2002). We have found, or improved, the distances, ages, and color excesses for 13 previously-known clusters. As one can see from Fig. 6, the number of clusters in the square studied, for which their main physical parameters are reliably evaluated, has increased from 11 in the catalog by Dias et al. (2002) to 35 in our study.

Therefore, to get a catalog of homogeneously-measured parameters of open clusters, it is necessary not only to search for new clusters and thoroughly investigate them, but also to recalculate the parameters of all known clusters using uniform raw datasets and a uniform and automated processing methodology. 
Acknowledgements. The work was supported by the Russian Foundation for Basic Research (grant no. 08-02-00381) and the President Grant NSH5290.2006.2. S. Koposov is supported by the DFG through SFB 439 and by a EARA-EST Marie Curie Visiting fellowship. This research has made use of the SAI Catalog Access Services, Sternberg Astronomical Institute, Moscow, Russia. This publication makes use of data products from the Two Micron All Sky Survey, which is a joint project of the University of Massachusetts and the Infrared Processing and Analysis Center/California Institute of Technology, funded by the National Aeronautics and Space Administration and the National Science Foundation. We thank for Hans-Walter Rix, Vasily Belokurov and Wilton Dias for the comments on earlier versions of this paper.

\section{References}

Babaud, J., Witkin, A. P., Baudin, M., \& Duda, R. O 1986, IEEE Trans. Pattern Anal. Mach. Intell., 8, 1, 26

Bica, E., Dutra, C. M., Soares, J., \& Barbuy, B. 2003a, A\&A, 404, 223

Bica, E., Dutra, C. M., \& Barbuy, B. 2003b, A\&A, 397, 177

Bhatt, B. C., Pandey, A. K., Mahra, H. S., \& Paliwal, D. C. 1994, Bull. Astr. Soc. India, 22, 291

Borissova, J., Pessev, P., Ivanov, V. D., et al. 2003, A\&A, 411, 83

Cardelli, J. A., Clayton, G. C., \& Mathis, J. S. 1989, ApJ, 345, 245

Carraro, G., Girardi, L., \& Marigo, P. 2002, MNRAS, 332, 705

Ciardullo, R. B., \& Demarque, P. 1979, In Problems of Calibration of Multicolor Photometric Systems, Dudley Observatory, 14, 317

Christian, C. A. 1980, AJ, 85, 700

Christian, C. A. 1981, ApJ, 246, 827
Dias, W. S., Alessi, B. S., Moitinho, A., \& Lépine, J. R. D. 2002, A\&A, 389, 871 and online version www. astro.iag.usp.br/ wilton/clusters.txt

Drake, A. J. 2005, A\&A, 435, 545

Dutra, C. M., Santiago, B. X., \& Bica, E. 2002, A\&A, 381, 219

Dutra, C. M., Bica, E., Soares, J., \& Barbuy, B. 2003, A\&A, 400, 533

Froebrich, D., Scholz, A., \& Raftery, C. L. 2007, MNRAS, 374, 399

Girardi, L., Bressan, A., Bertelli, G., \& Chiosi, C. 2000, A\&AS, 141, 371

Girardi, L., Bertelli, G., Bressan, A., et al. 2002, A\&A, 391, 195

Ivanov, V. D., Borissova, J., Pessev, P., Ivanov, G. R., \& Kurtev, R. 2002, A\&A, 394, L1

Koposov, S., Glushkova, E., \& Zolotukhin, I. 2005, Astron. Nachr., 326, 597

Koposov, S., de Jong, J. T. A., Belokurov, V., et al. 2007, ApJ, 669, 337

Koposov, S., et al. 2008, ApJ, accepted [arXiv:0706.2687]

Koposov, S. E., Bartunov, O., \& Karpov, S. 2007, Highlights Astron., 14, 586

Kronberger, M., Teutsch, P., Alessi, B., et al. 2006, A\&A, 447, 921

Lata, S., Mohan, V., \& Sagar, R. 2004, Bull. Astron. Soc. India, 32, 371

Lindenberg, T. 1998, Inter. J. Comp. Vis., 30, 2, 79

Loktin, A. V., Gerasimenko, T. P., \& Malisheva, L. K. 2001, Astron. Astrophys. Trans., 20, 607

Paunzen, E., \& Mermilliod, J.-C. http://www .univie.ac.at/webda/

Schmidt-Kaler Th. 1982, in Landolt/Bornstein, Numerical Data and Functional Relationship in Science and Technology, ed. K. Scaifers, H. H. Voigt, New series, Group VI, $2 b$ (Berlin: Springer-Verlag), 14

Skrutskie, M. F., Cutri, R.M., Stiening, R., et al. 2006, AJ, 131, 1163

Surdin, V. G. 2000, Formación estelar, Editorial URSS, Moscú, 272

Vallenari, A., Richichi, A., Carraro, G., \& Girardi, L. 1999, A\&A, 349, 825

Zolotukhin, I., Koposov, S., \& Glushkova, E. 2006, Astronomical Data Analysis Software and Systems XV, ASP Conf. Ser. 351, 240 
S. E. Koposov et al.: Automated search for Galactic star clusters in large multiband surveys. I., Online Material p 1
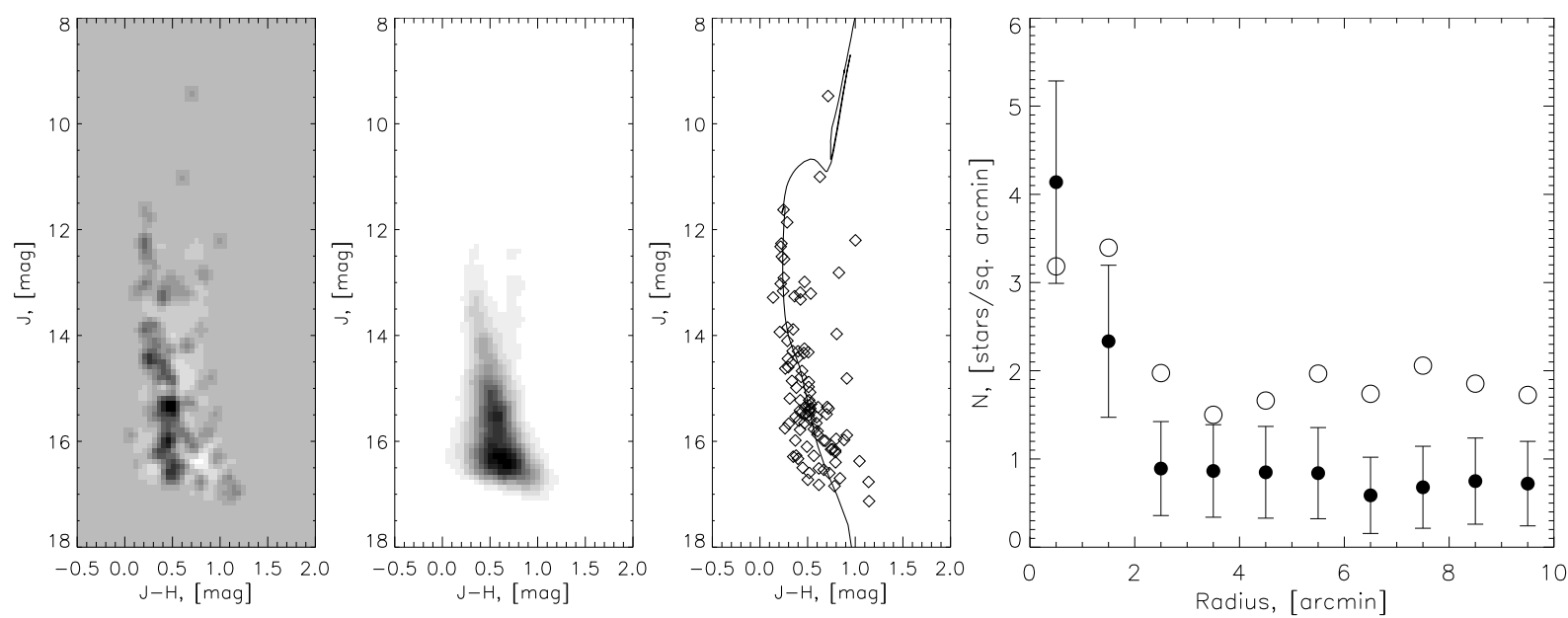

Fig. 7. First and second columns: Hess diagram of the Koposov 10 cluster and Hess diagram of the background. Third column: CMD diagram of the stars within $2^{\prime}$ from the center of the Koposov 10 with the fitted isochrone. Fourth column: radial density distribution for Koposov 10 , the symbols used are the same as in Fig. 5.
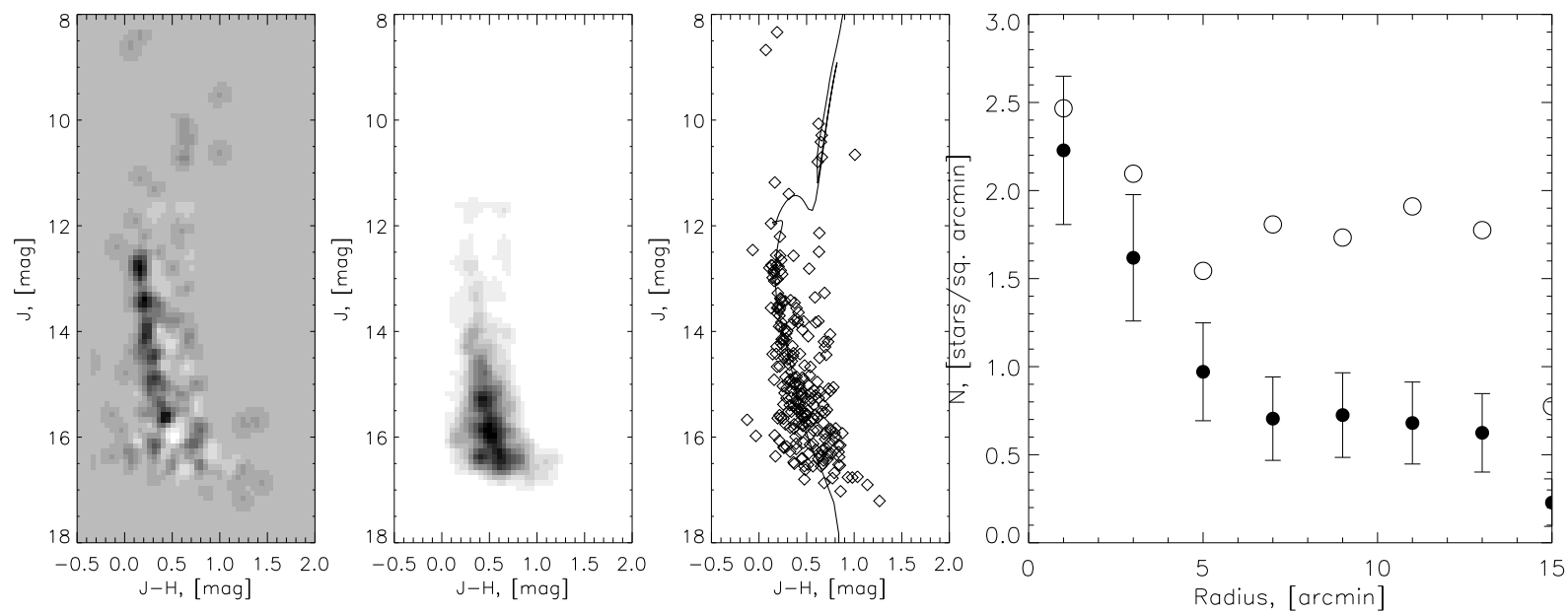

Fig. 8. First and second columns: Hess diagram of the Koposov 12 cluster and Hess diagram of the background. Third column: CMD diagram of the stars within $4^{\prime}$ from the center of the Koposov 12 with the fitted isochrone. Fourth column: Radial density distribution for Koposov 12, the symbols used are the same as in Fig. 5.
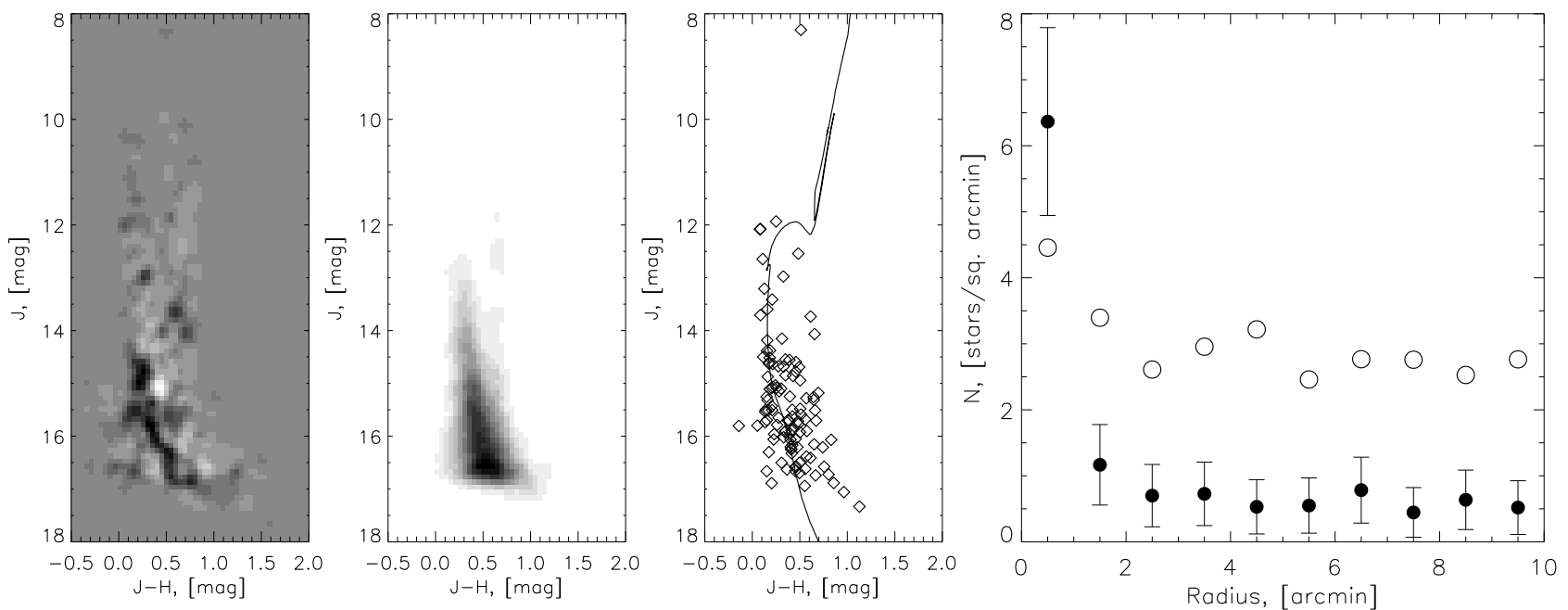

Fig. 9. First and second columns: Hess diagram of the Koposov 27 cluster and Hess diagram of the background. Third column: CMD diagram of the stars within $2^{\prime}$ from the center of the Koposov 27 with the fitted isochrone. Fourth column: Radial density distribution for Koposov 27 , the symbols used are the same as in Fig. 5. 
S. E. Koposov et al.: Automated search for Galactic star clusters in large multiband surveys. I., Online Material p 2
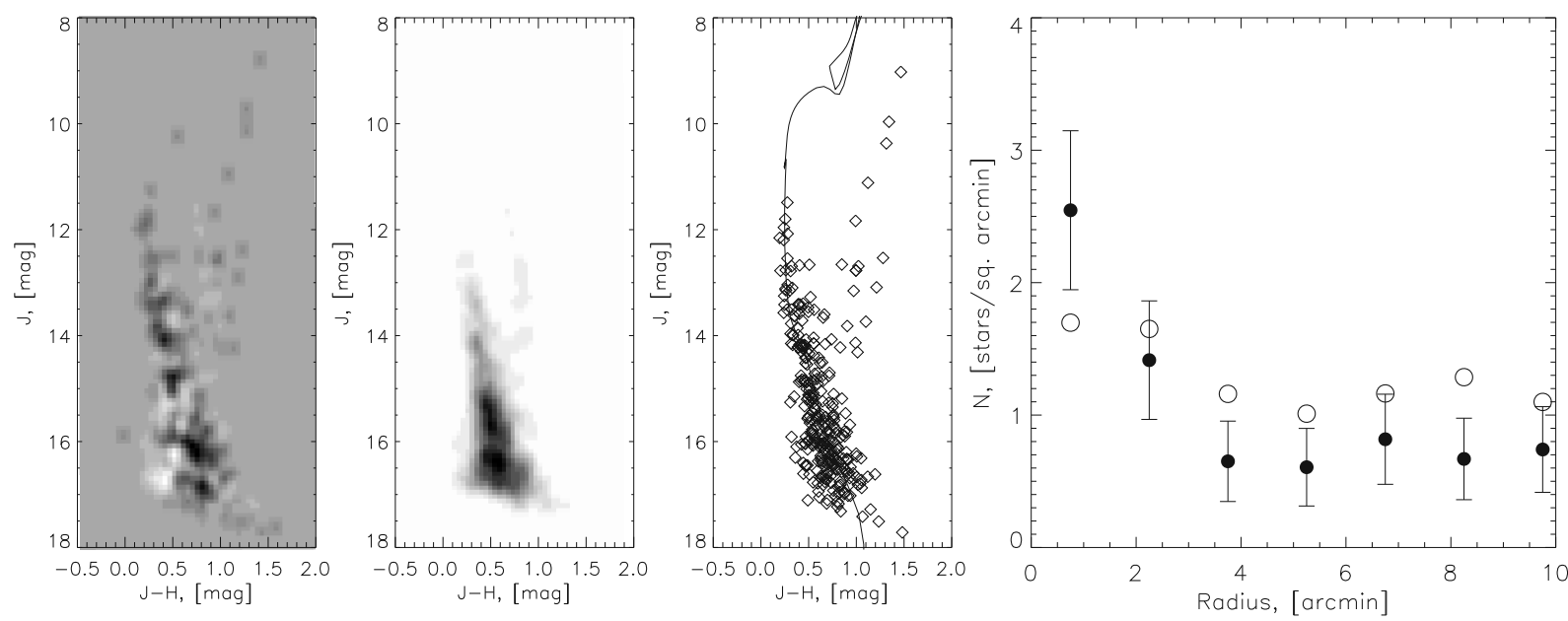

Fig. 10. First and second columns: Hess diagram of the Koposov 36 cluster and Hess diagram of the background. Third column: CMD diagram of the stars within 4' from the center of the Koposov 36 with the fitted isochrone. Fourth column: radial density distribution for Koposov 36 , the symbols used are the same as in Fig. 5.
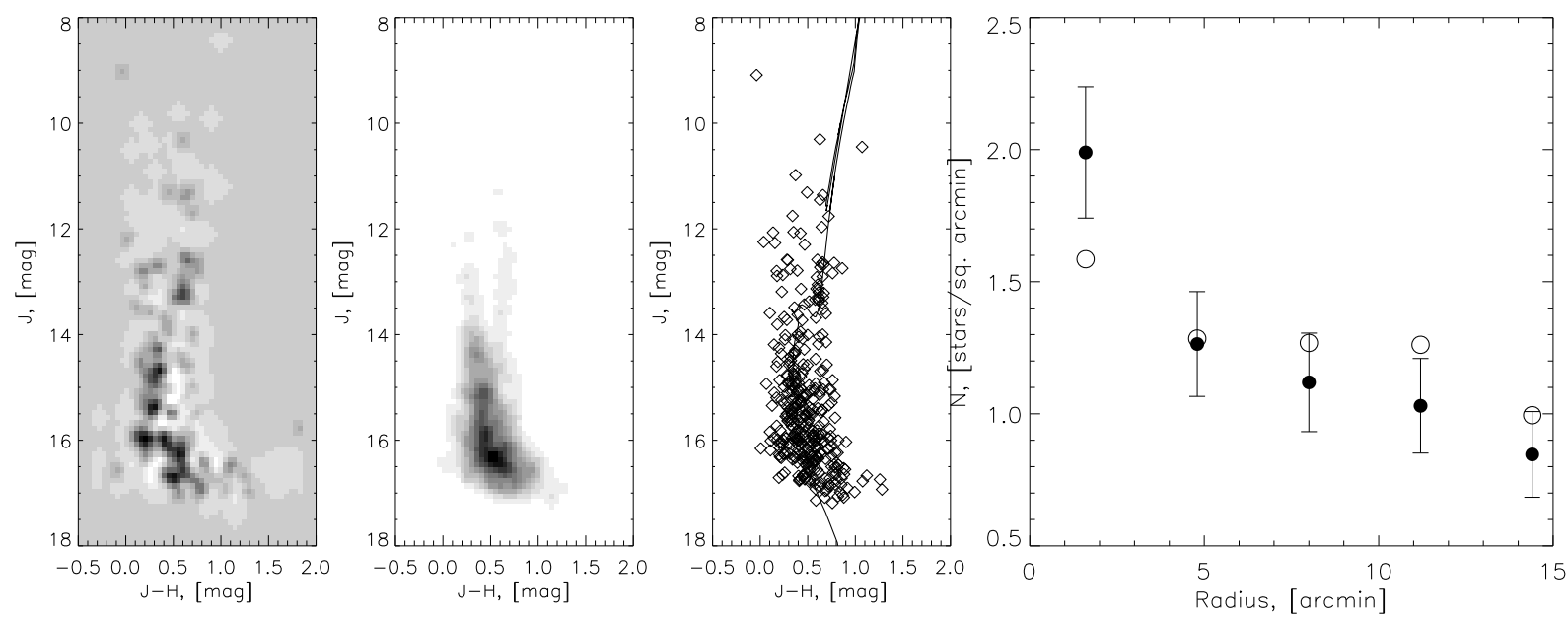

Fig. 11. First and second columns: Hess diagram of the Koposov 43 cluster and Hess diagram of the background. Third column: CMD diagram of the stars within 4' from the center of the Koposov 43 with the fitted isochrone. Fourth column: radial density distribution for Koposov 43 , the symbols used are the same as in Fig. 5.
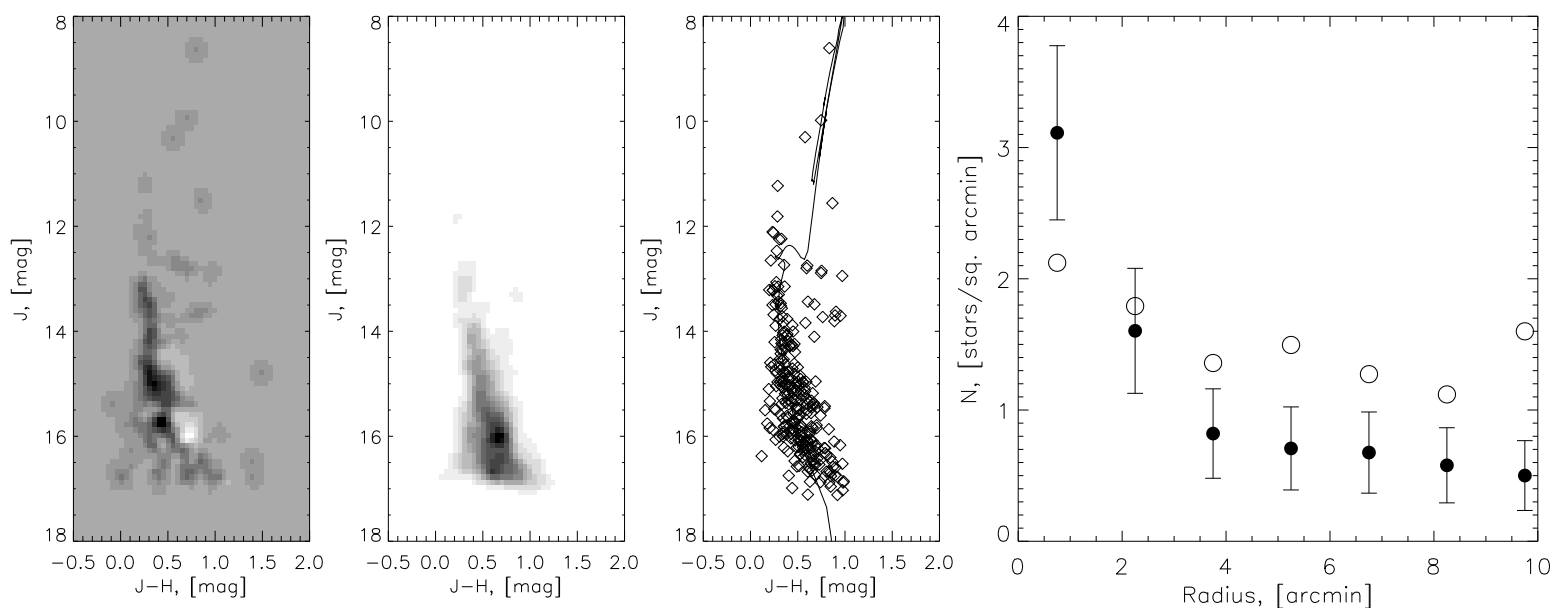

Fig. 12. First and second columns: Hess diagram of the Koposov 49 cluster and Hess diagram of the background. Third column: CMD diagram of the stars within $4^{\prime}$ from the center of the Koposov 49 with the fitted isochrone. Fourth column: radial density distribution for Koposov 49 , the symbols used are the same as in Fig. 5. 
S. E. Koposov et al.: Automated search for Galactic star clusters in large multiband surveys. I., Online Material p 3
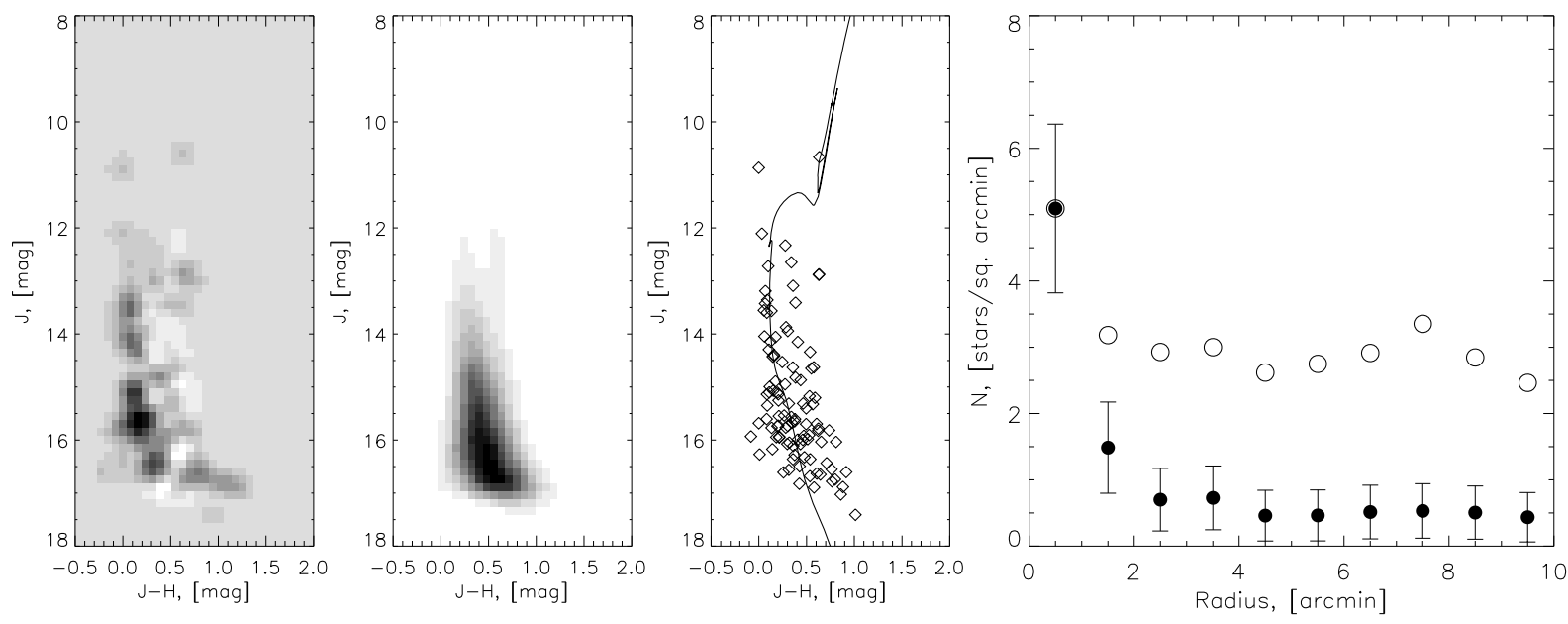

Fig. 13. First and second columns: Hess diagram of the Koposov 53 cluster and Hess diagram of the background. Third column: CMD diagram of the stars within 4' from the center of the Koposov 53 with the fitted isochrone. Fourth column: radial density distribution for Koposov 53, the symbols used are the same as in Fig. 5.
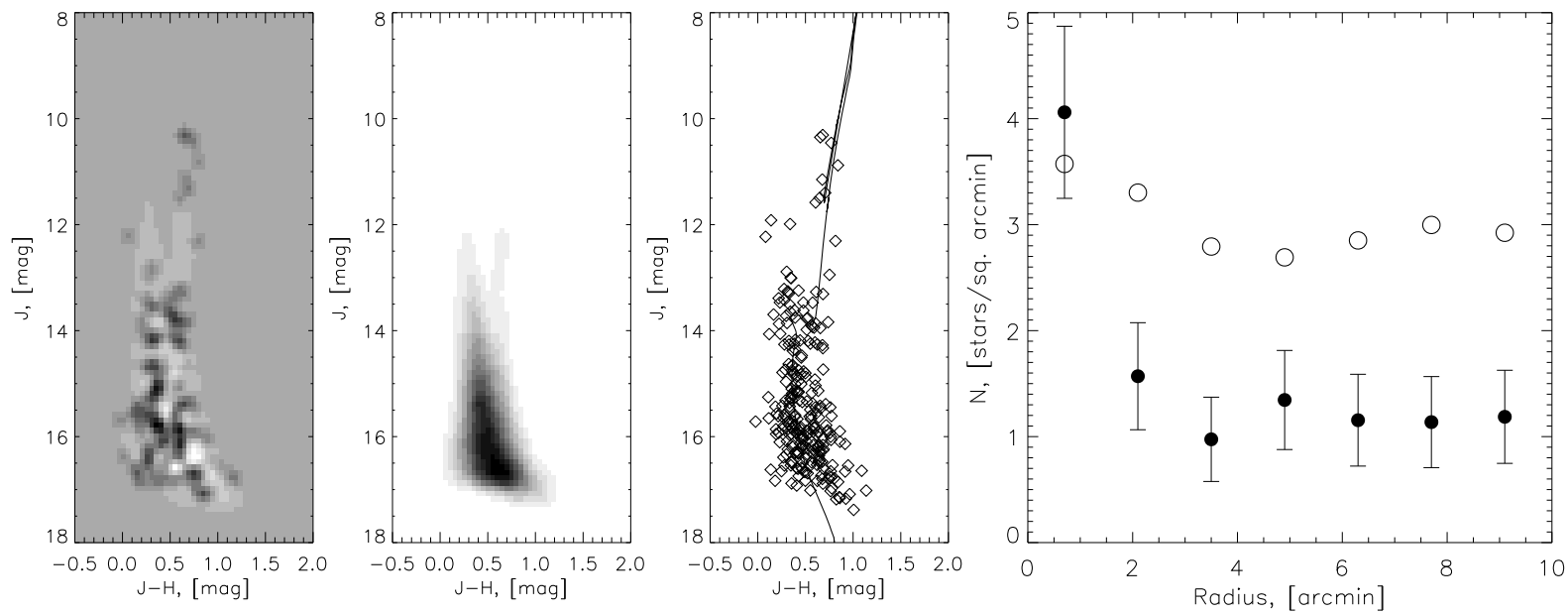

Fig. 14. First and second columns: Hess diagram of the Koposov 62 cluster and Hess diagram of the background. Third column: CMD diagram of the stars within $3^{\prime}$ from the center of the Koposov 62 with the fitted isochrone. Fourth column: radial density distribution for Koposov 62 , the symbols used are the same as in Fig. 5.
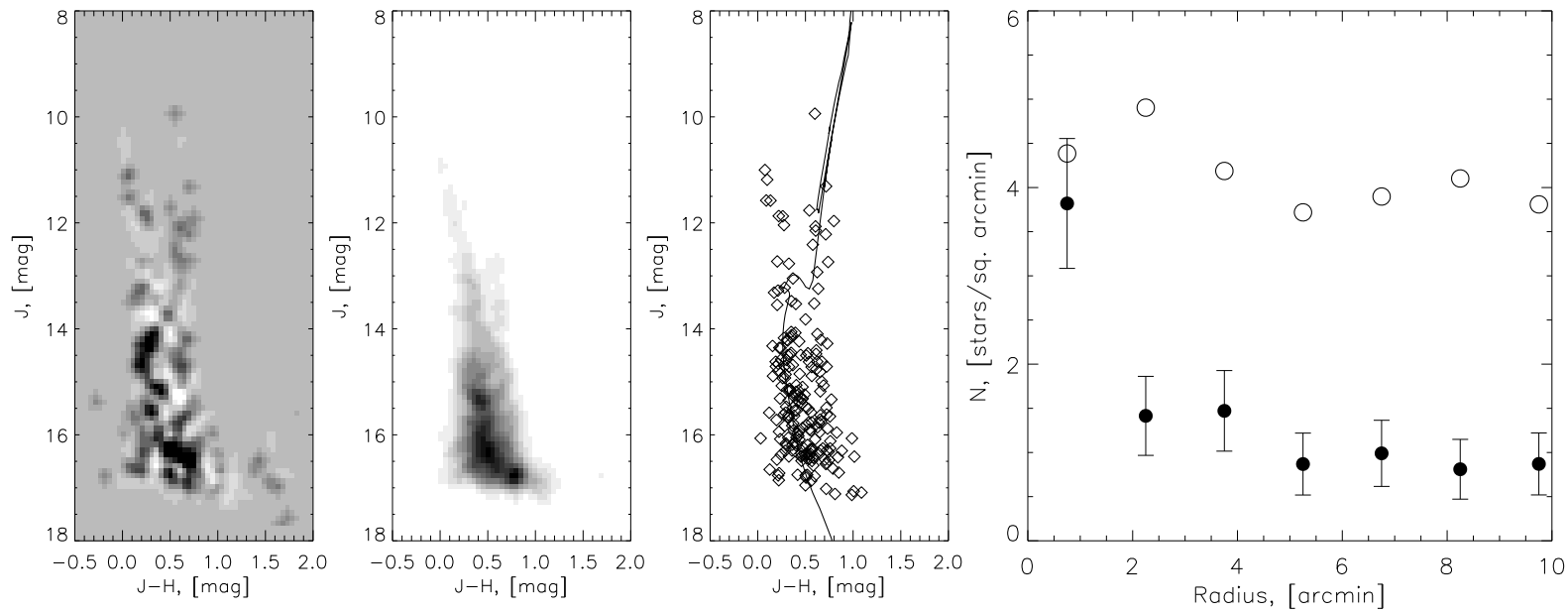

Fig. 15. Koposov 63 open cluster. First and second columns: Hess diagram of the Koposov 63 cluster and Hess diagram of the background. Third column: CMD diagram of the stars within 2'.5 from the center of the Koposov 63 with the fitted isochrone. Fourth column: radial density distribution for Koposov 63, the symbols used are the same as in Fig. 5. 
S. E. Koposov et al.: Automated search for Galactic star clusters in large multiband surveys. I., Online Material p 4
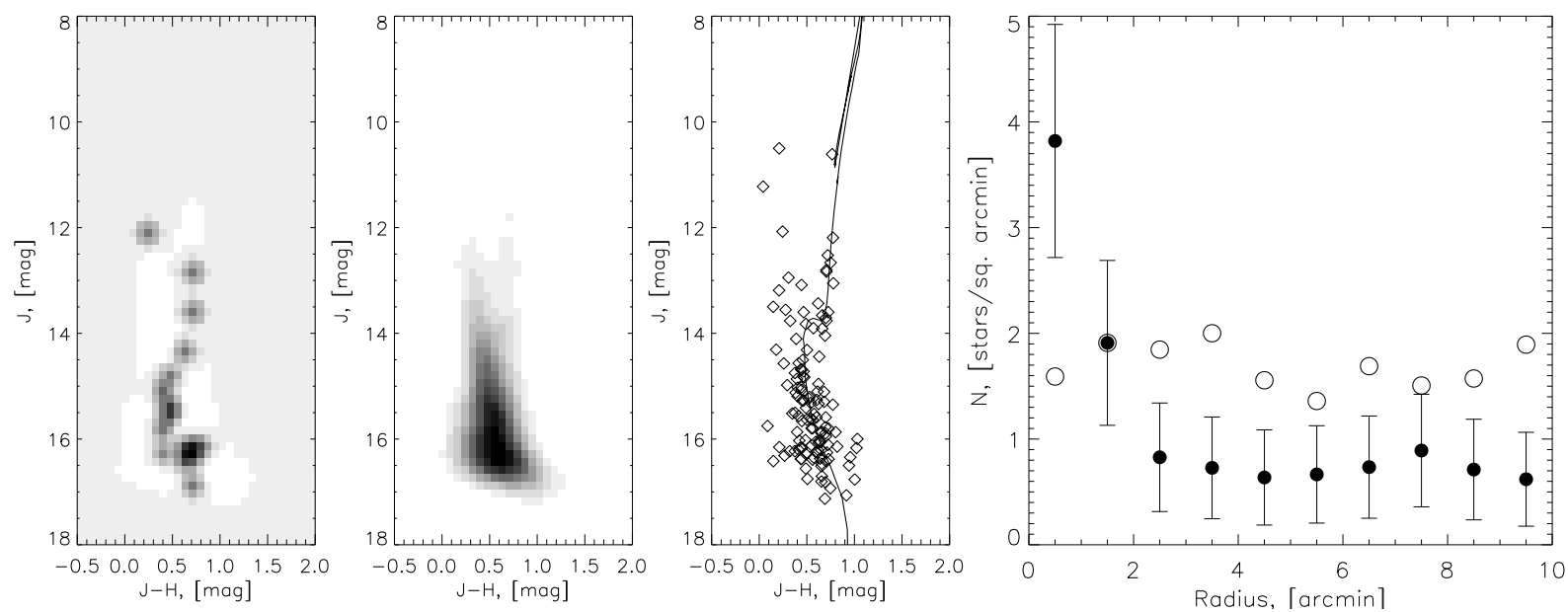

Fig. 16. Koposov 77 open cluster. First and second columns: Hess diagram of the Koposov 77 cluster and Hess diagram of the background. Third column: CMD diagram of the stars within $2^{\prime} .5$ from the center of the Koposov 77 with the fitted isochrone. Fourth column: Radial density distribution for Koposov 77, the symbols used are the same as in Fig. 5.
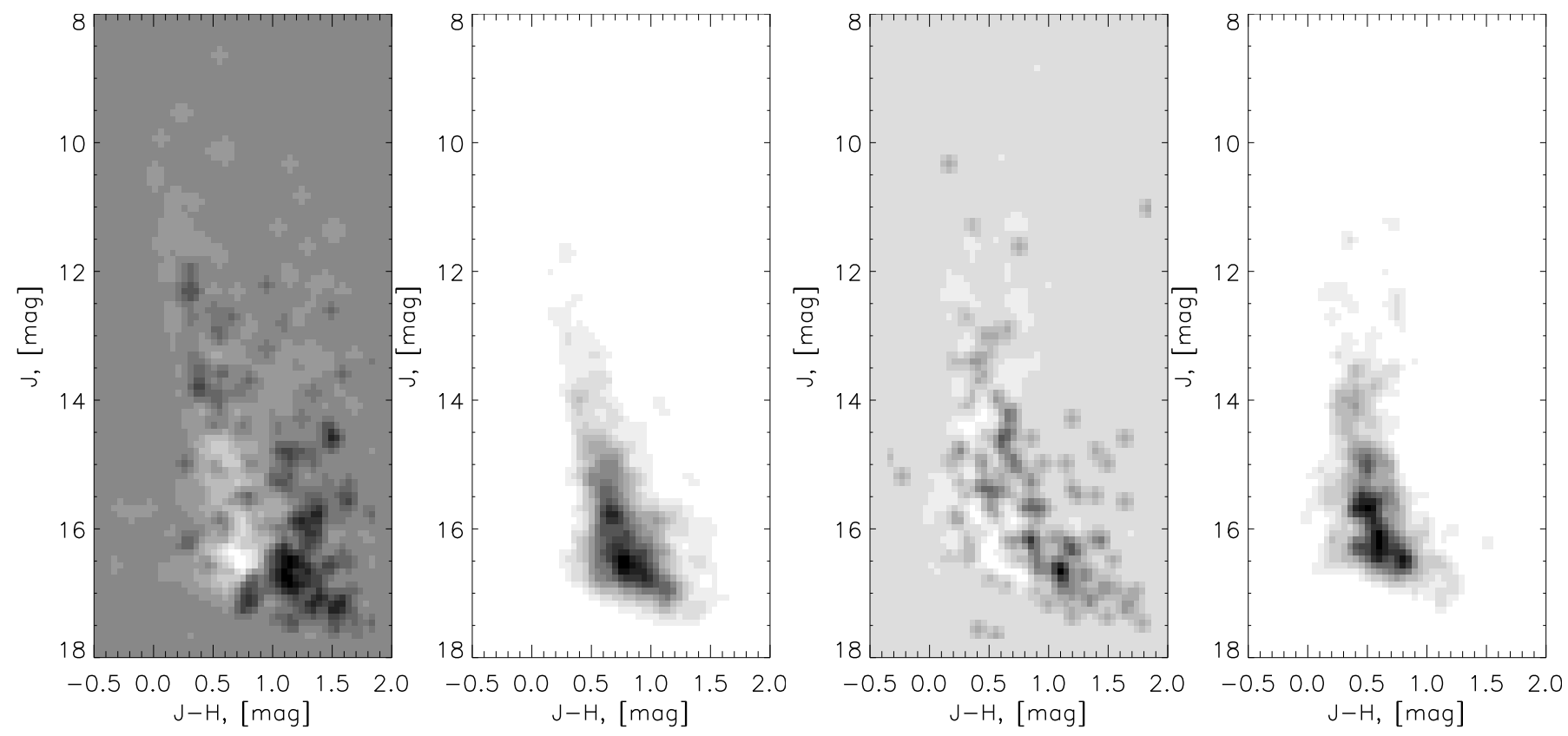

Fig. 17. Left panel: Hess diagram of the IR-embedded cluster Koposov 7 and Hess diagram of the background. Right panel: Hess diagram of the IR-embedded cluster Koposov 41 and Hess diagram of the background. 
S. E. Koposov et al.: Automated search for Galactic star clusters in large multiband surveys. I., Online Material p 5
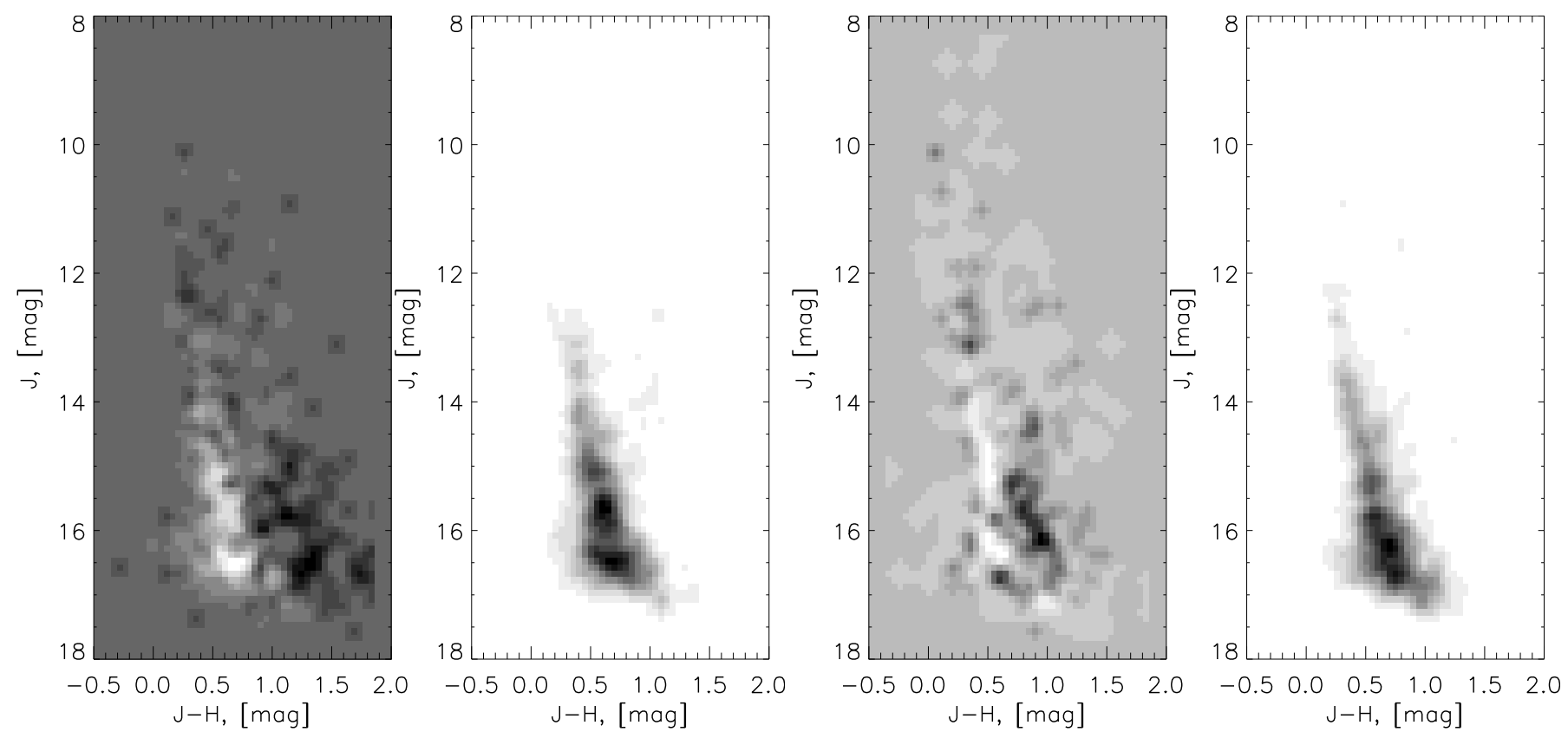

Fig. 18. Left panel: Hess diagram of the IR-embedded cluster Koposov 58 and Hess diagram of the background. Right panel: Hess diagram of the IR-embedded cluster Koposov 82 and Hess diagram of the background.

Table 3. Comparison of physical parameters of the clusters: Dias catalog and Paunzen-Mermilliod database (DPM) vs. present study (KGZ).

\begin{tabular}{|c|c|c|c|c|c|c|c|c|}
\hline Name & $\begin{array}{l}\text { RA(J2000) } \\
\text { h:m:s }\end{array}$ & $\begin{array}{l}\operatorname{Dec}(\mathrm{J} 2000) \\
\text { d:m:s }\end{array}$ & $\begin{array}{c}D_{\mathrm{DPM}} \\
\mathrm{pc}\end{array}$ & $\begin{array}{c}D_{\mathrm{KGZ}} \\
\mathrm{pc}\end{array}$ & $\begin{array}{c}E(B-V)_{\mathrm{DPM}} \\
\text { mag }\end{array}$ & $\begin{array}{c}E(B-V)_{\mathrm{KGZ}} \\
\mathrm{mag}\end{array}$ & $\begin{array}{c}\text { Age }_{\text {DPM }} \\
\log (y r)\end{array}$ & $\begin{array}{l}\mathrm{Age}_{\mathrm{KGZ}} \\
\log (\mathrm{yr})\end{array}$ \\
\hline Basel 4 & $05: 48: 54.9$ & $+30: 11: 08$ & 3000 & 2750 & 0.45 & 0.57 & 8.30 & 8.25 \\
\hline Berkeley 17 & $05: 20: 29.6$ & $+30: 34: 33$ & 2700 & 2400 & 0.58 & 0.30 & 10.00 & 10.00 \\
\hline Berkeley 19 & $05: 24: 02.8$ & $+29: 34: 16$ & 4831 & 3000 & 0.40 & 0.61 & 9.49 & 9.25 \\
\hline Berkeley 21 & $05: 51: 47.4$ & $+21: 48: 31$ & 5000 & 5150 & 0.76 & 0.51 & 9.34 & 9.35 \\
\hline Berkeley 69 & $05: 42: 22.6$ & $+22: 50: 01$ & 2860 & 2900 & 0.65 & 0.45 & 8.95 & 9.00 \\
\hline Berkeley 71 & $05: 40: 56.7$ & $+32: 16: 33$ & 3900 & 2450 & 0.85 & 0.91 & 8.80 & 8.80 \\
\hline Berkeley 72 & $05: 50: 17.6$ & $+22: 14: 59$ & & 3500 & & 0.43 & & 8.65 \\
\hline Czernik 21 & $05: 26: 41.0$ & $+36: 00: 49$ & & 2300 & & 0.72 & & 9.55 \\
\hline Czernik 23 & 05:50:03.6 & +28:53:41 & & 2500 & & 0.38 & & 8.45 \\
\hline Czernik 24 & $05: 55: 24.6$ & $+20: 53: 11$ & & 4600 & & 0.26 & & 9.40 \\
\hline DC 8 & $06: 09: 21.3$ & $+31: 13: 54$ & & 2100 & & 0.72 & & 9.00 \\
\hline IC 2157 & 06:04:41.9 & $+24: 06: 01$ & 2040 & 2400 & 0.548 & 0.58 & 7.800 & $<7.6$ \\
\hline King 8 & $05: 49: 19.0$ & $+33: 37: 38$ & 6403 & 3100 & 0.580 & 0.44 & 8.618 & 9.05 \\
\hline Kronberger 1 & $05: 28: 22.0$ & $+34: 46: 24$ & 1900 & 800 & 0.52 & 0.43 & 7.5 & 8.10 \\
\hline NGC 1893 & $05: 22: 53.7$ & $+33: 26: 17$ & 6000 & & 0.45 & & 6.48 & \\
\hline NGC 1907 & 05:28:10.7 & +35:19:44 & 1800 & 1300 & 0.52 & 0.51 & 8.5 & 8.60 \\
\hline NGC 1931 & $05: 31: 25.9$ & $+34: 12: 50$ & 3086 & 1000 & 0.738 & 1.97 & 7.002 & $<7.0$ \\
\hline NGC 1960 & 05:36:19.6 & $+34: 07: 27$ & 1330 & 1050 & 0.22 & 0.19 & 7.4 & $<7.5$ \\
\hline NGC 2099 & $05: 52: 18.4$ & $+32: 33: 03$ & 1383 & 1300 & 0.302 & 0.27 & 8.540 & 8.60 \\
\hline NGC 2129 & $06: 01: 10.5$ & +23:19:34 & 2200 & 1950 & 0.80 & 0.82 & 7.00 & 7.10 \\
\hline NGC 2158 & 06:07:27.8 & $+24: 05: 53$ & 5071 & 3300 & 0.360 & 0.34 & 9.023 & 9.30 \\
\hline Pismis 27 & $06: 10: 53.8$ & $+20: 36: 26$ & & 1000 & & 0.68 & & $<7.7$ \\
\hline Stock 8 & 05:28:08.8 & $+34: 25: 53$ & 1821 & 900 & 0.445 & 1.21 & 7.056 & $<7.5$ \\
\hline NGC 1912 & $05: 28: 41.6$ & $+35: 48: 34$ & 1400 & 1000 & 0.25 & 0.38 & 8.5 & 8.30 \\
\hline NGC 2168 & 06:09:00.0 & $+24: 21: 00$ & 912 & 900 & 0.20 & 0.19 & 8.25 & 7.95 \\
\hline
\end{tabular}

